EPJ Web of Conferences 59, 01007 (2013)

DOI: $10.1051 /$ epjconf/20135901007

(C) Owned by the British Crown, published by EDP Sciences, 2013

\title{
Overview of recent AWE fusion-related studies, experiments and facilities
}

\author{
P.D. Roberts
}

AWE, Aldermaston, Reading, Berkshire, England RG7 4PR, UK

\begin{abstract}
The presentation will describe the current status of modelling short and long pulse laser irradiation and its application to inertial fusion designs. Recent results will be described which give confidence in the modelling in specific regimes. An update will be given of the AWE ORION laser facility and the availability planned for academic access.
\end{abstract}

\section{AWE PERSPECTIVE}

Following the demonstration of the Laser in 1960, many laboratories considered the possibility of achieving fusion through laser heating of a DT plasma. The seminal paper by Nuckolls [1] in 1972 identified the significance of compression and the possibility of achieving ignition with only kilojoules of laser energy using a temporally shaped laser pulse. At AWE this stimulated the writing of the 1D and 2D simulation codes, NYM, to evaluate the concept independently. In 1973 AWE left the United Kingdom Atomic Energy Authority (UKAEA) and became part of the Ministry of Defence (MoD). During that decade the production of X-rays associated with laser irradiation, particularly of high-Z targets, became a topic of particular study as it offered the near-term prospect of studying aspects of weapon physics. The proposal was to use a high-Z hohlraum to both enhance laser absorption and to create a thermal radiation field. At this time the concept was classified and little was published. The conversion to thermal X-rays also offered a route to achieving fusion but higher energy seemed to be needed so experiments were proposed using the environment of an underground nuclear test. In parallel, the case for a TW Nd laser was accepted and the HELEN laser was opened in 1979. During the 1980s HELEN confirmed the approach of using high power lasers to study material properties at temperatures of around $100 \mathrm{eV}$ together with the ability to study radiation transport and radiation hydrodynamics. The available energy $(2 \times 1 \mathrm{kj}, 1 \mathrm{~ns}, 1 \omega)$ meant that quantitative measurements of these more integrated aspects were difficult and the hohlraum temperatures achieved were somewhat lower than originally anticipated, due primarily to non-LTE effects. Subsequently temperatures in excess of $500 \mathrm{eV}$ were demonstrated using CPA technology and conversion to $2 \omega$. On the fusion side, a UK experiment in 1982 was successful and gave some confidence in key physics aspects. In the $1990 \mathrm{~s}$ the UK ceased underground testing which gave greater impetus to the use of high energy lasers however the case for a $100 \mathrm{TW}$ laser proved unaffordable. In the same decade (1993) AWE left the MoD and became contractorised under an arrangement known as GOCO (Government Owned Contractor Operated). During the $00 \mathrm{~s}$ decade this contract was re-competed and the contractor changed, in the same decade the case for ORION was accepted and construction started in 2006. This was coupled with an agreement between US and UK governments which provided access to NIF. The overall strategy is to use ORION primarily for material properties studies and NIF for more integrated experiments. We have also used the OMEGA laser at Rochester for a variety of studies, some of which will be described in this paper.

This is an Open Access article distributed under the terms of the Creative Commons Attribution License 2.0, which permits unrestricted use, distribution, and reproduction in any medium, provided the original work is properly cited. 


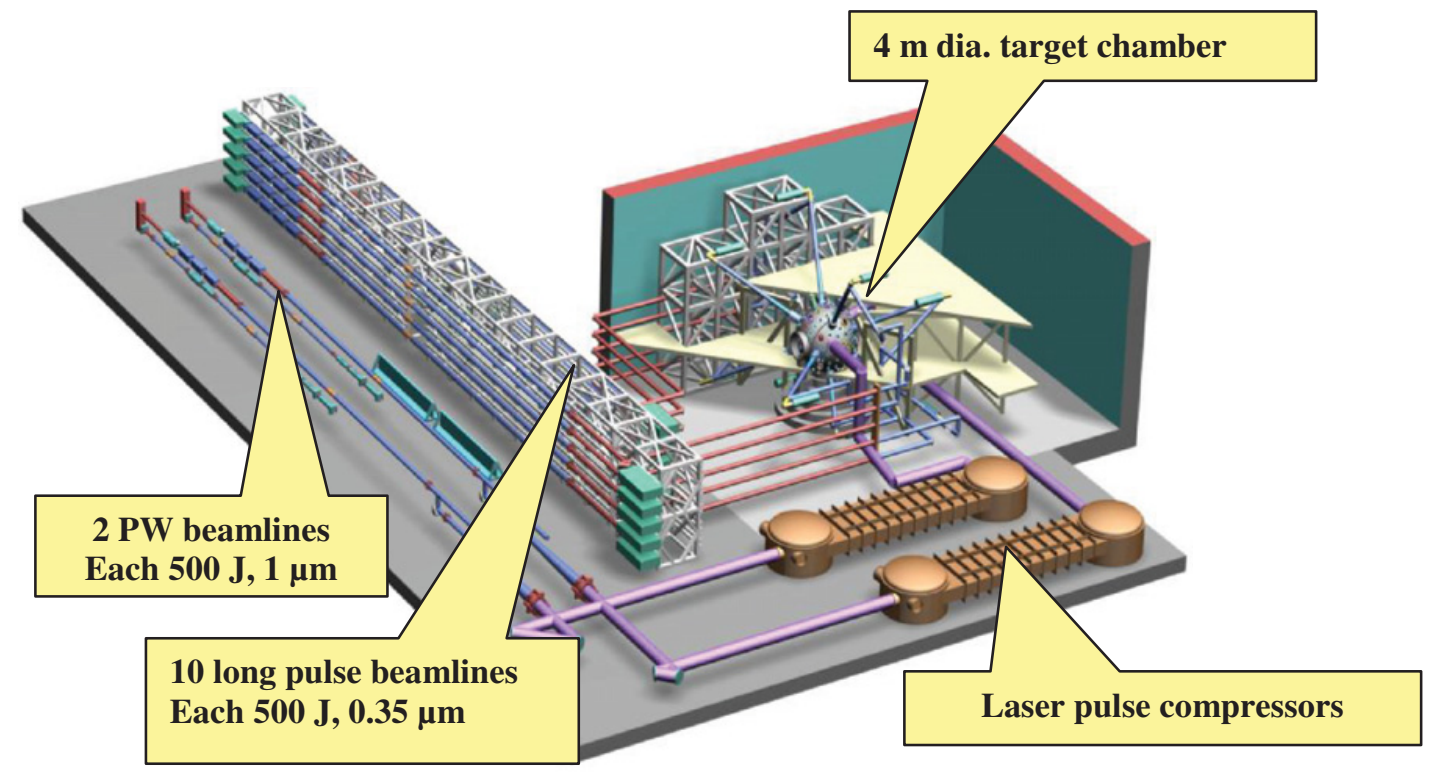

Figure 1. ORION layout and key features.

Finally in this last decade, ignition has become a real possibility and we have started to re-examine this approach and some of the implications that would follow from success (for example Inertial Fusion Energy, IFE).

\section{ORION - STATUS AND SCHEDULE}

Figure 1, below, shows the layout of the ORION laser, including the 10 long pulse ( $1 \mathrm{~ns})$ 'compression' beams and the 2 short pulse (1 ps) 'heating' beams.

The project was approved in 2005 and construction began a year later. By the end of 2010 all the systems and sub-systems had been installed and a key milestone achieved which required the firing of one long and one short pulse into the target chamber. By the end of March 2012 it is expected that all beams will have been commissioned and synchronized. A further 12 months is then scheduled to demonstrate the experimental capability and this requires shock compressing an aluminium target by a factor of 2 and simultaneously heating it to $500 \mathrm{eV}$. At this point the laser will effectively be open for business, which includes making $15 \%$ of the times available to the academic community.

\section{NUMERICAL SIMULATION}

Two numerical codes are currently in use for modeling capsule implosion, NYM - the legacy code and CORVUS a more recent code. The main deficiency of NYM is that is has only a simple arbitrary Lagrange-Eulerian treatment for the hydrodynamics which means material cannot be advected across interfaces. CORVUS has a more complete implementation which allows integrated modelling of a capsule in a laser-driven hohlraum. NYM, however, has much physics in it which has developed over many years so CORVUS is not yet a complete replacement. To study laser-plasma interactions in detail we use the EPOCH particle-in-cell code, developed in collaboration with Warwick University. Such interactions also require the modeling of electron transport in a self - consistent manner which allows for long range forward currents and a lower energy return current to maintain charge neutrality. This aspect is modeled in THOR which uses a Monte Carlo approach. 


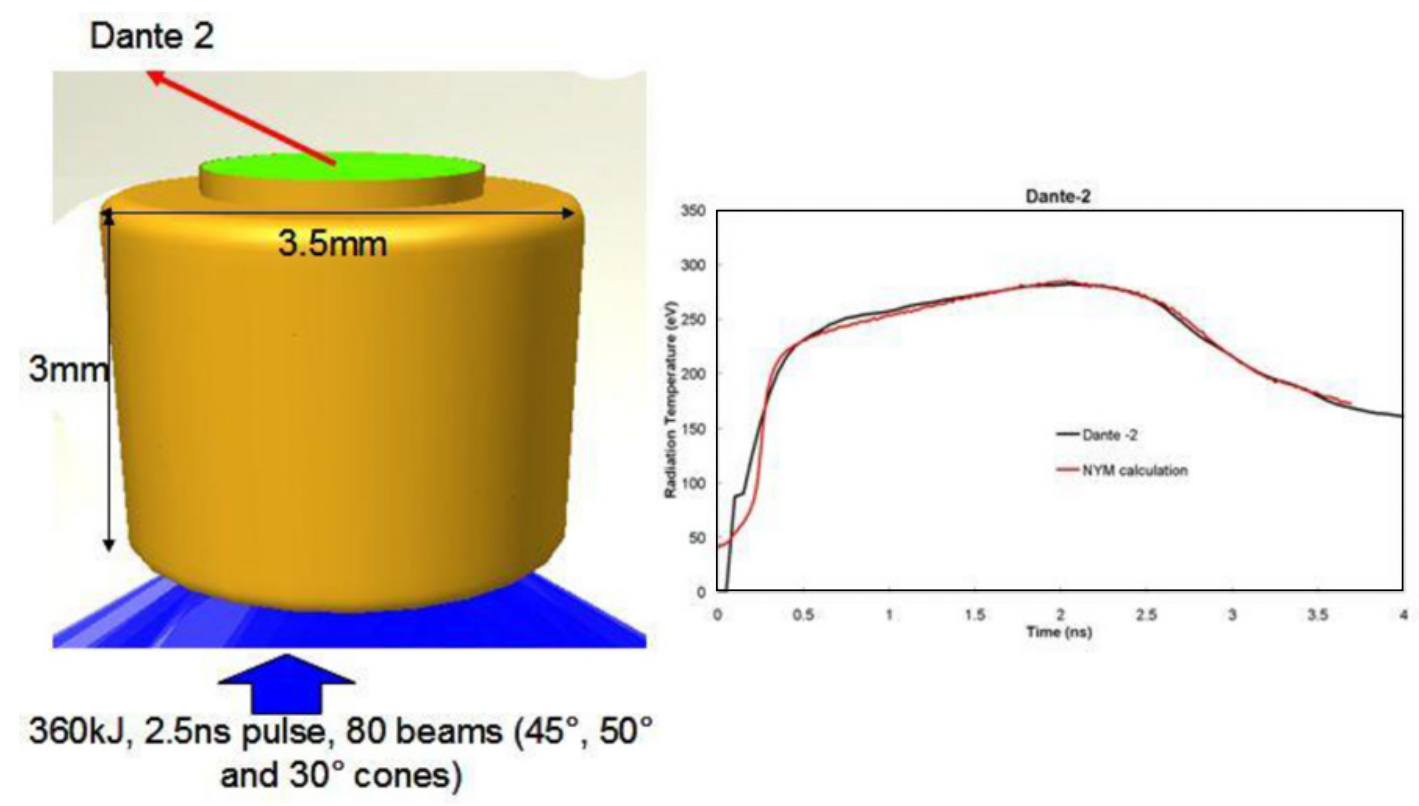

Figure 2. Hohlraum validation.

\section{MODEL VALIDATION}

A large number of hohlraum experiments have been conducted over the years and the models developed and validated against this data. Figure 2 gives an example of a recent hohlraum experiment fielded at the National Ignition Facility (NIF).

To model the temperature measured by the DANTE 2 diagnostic requires not only careful modeling of the hohlraum interaction but also attention must be paid to what the diagnostic is actually looking at. In this case we have an $\mathrm{SiO}_{2}$ window which makes interpretation somewhat simpler.

To validate the ability to model, and control, the implosion symmetry of a capsule in a hohlraum a number of experiments have been undertaken [2] at the University of Rochester using the OMEGA laser. Asymmetry has been introduced in various ways; changing the irradiation beam balance, changing the material at one end of the hohlraum, moving the capsule and introducing baffles. To model these extreme plasma flows the NYM code was used linked at a late time to the PETRA Eulerian code. Although PETRA does not have all the physics that NYM does, by linking at a late time, the laser pulse has terminated and the hydrodynamic flow is committed. The comparison between calculation and experiment if very good although a reduction of about $8 \%$ in the laser energy is required to match the implosion timing. This is consistent with energy absorbed in the debris shields and a direct measure of the laser energy incident though the hohlraum entrance holes is not available.

\section{FUSION APPLICATIONS}

An approach that allows rapid assessment of parameter sensitivity and large numbers of runs to be undertaken quickly was implemented in the early days of NYM. The model is 1D, spherical, and allows the laser light to enter the mesh some way inside the problem and is tracked outwards. The true wall area is preserved in the 1D geometry and direct radiation loss from the problem simulates that from the hohlraum entrance holes. Figure 3 shows a typical simulation for an early LLNL point design and displays good agreement with a much more sophisticated approach. 

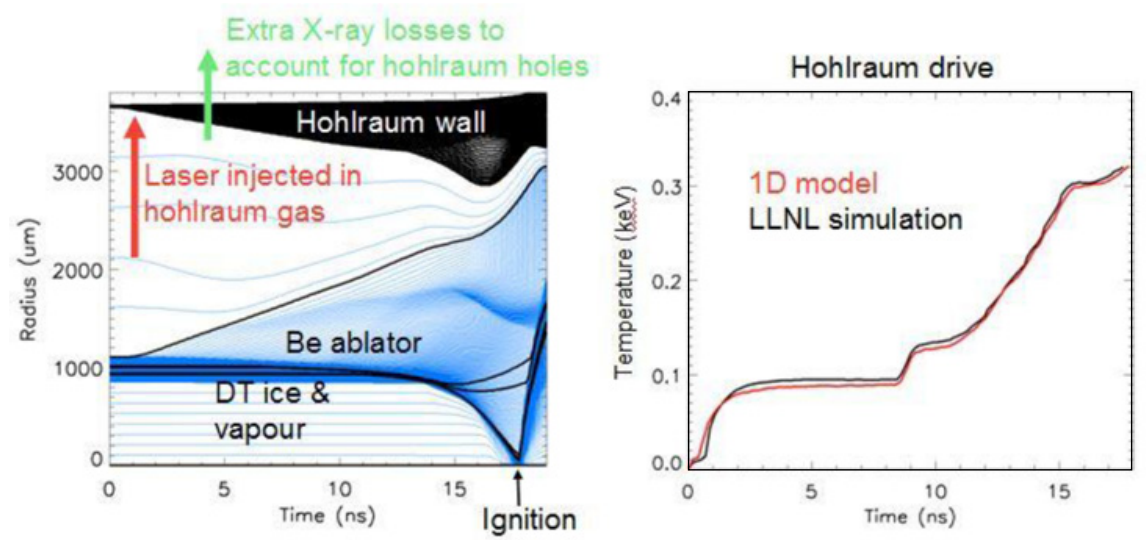

True wall area conserved

Figure 3. Simple 1D model for parameter studies.

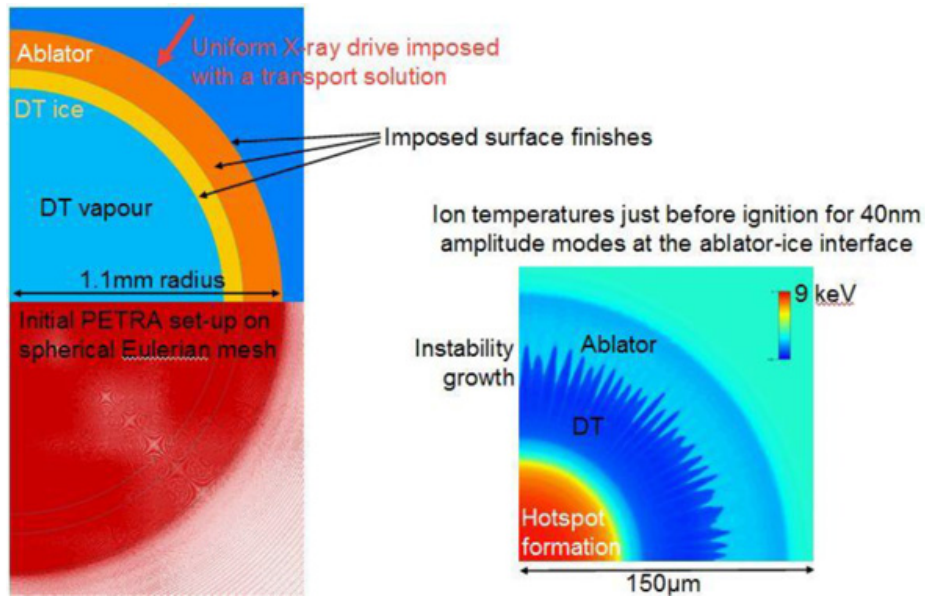

Figure 4. Calculation of perturbation growth.

In the same vein, we have used an isobaric model of hot spot ignition and burn to understand the sensitivity of the propagation physics from hot spot to cold fuel. We set up the initial conditions in the hot spot as a $\rho \mathrm{R}$ of $0.3 \mathrm{~g} / \mathrm{cm}^{2}$ and an initial temperature of $10 \mathrm{keV}$. We took a typical NIF ignition capsule, kept the fuel mass constant, and reduced the cold fuel density while increasing its temperature to maintain isobaric conditions. We found we could reduce the cold fuel density by about a factor of 3 and still get propagation while not overly perturbing the system energetics. Of course the gain drops as the system $\rho \mathbf{R}$ falls but still provides a modest gain.

Rayleigh-Taylor instability is a potential barrier to achieving ignition and high gain and we have started to study this using an Eulerian 2D code, based on PETRA, that tracks the implosion of the capsule using a 1D Lagrangian re-map. In figure 4 we show a calculation of an imposed $40 \mathrm{~nm}$ perturbation and its amplitude at the point of hot spot ignition. 
Further validation of the model is required but it does demonstrate the ability to simulate such instabilities and also the potential concern over whether they can be adequately controlled given that any perturbation, not just geometric, can seed the instability.

\section{SUMMARY}

This is undoubtedly an exciting time with ignition credible in the near future and ORION operational next year. The availability of $15 \%$ of ORION time to the academic community should further enhance its value and the range of science that will be studied. We have collaborated with US colleagues in a range of high energy density physics studies on NIF and have had very good success. Of course these experiments are typically not as demanding as for ignition but nevertheless given suitable attention to detail we have modeled the data well and have not encountered any unexpected physics. We are becoming increasingly engaged with the ignition challenge and have demonstrated that many of the required tools are now in place.

C. Sorce, G. Glendinning, T. Goldsack, J. Foster, K. Widman, J. Kline, P. Keiter, P. Rosen, D. Hoarty, P. Thompson, T. Bett, C. Danson, D. Schmidt, N. Sircombe, S. Hughes, J. Morton, B. Thomas, M. Stevenson, P. Graham, W. Garbett, B. Williams, S. McAlpin, K. Vaughan, M. Taccetti, K. Mussack, G. Magelsson, B. Peterson, N. Lanier, J. Workman, W. Hsing

\section{References}

[1] J. Nuckolls et al. Nature, 239, 139 (1972)

[2] K. Vaughan, S. McAlpin, J. M. Foster, R. M. Stevenson, S. G. Glendinning, and C. Sorce, Phys. Plasmas 17, 056316 (2010) 